DOI: 10.12731/2227-930X-2021-11-1-45-58

УДК 004.722

\title{
АВТОМАТИЗИРОВАННАЯ СИСТЕМА УПРАВЛЕНИЯ КАМНЕДРОБИЛЬНОЙ УСТАНОВКОЙ
}

\author{
Мясников В.И., Морохин Д.В., \\ Иванов А.В., Иванов Р.А.
}

Вопросы автоматизачии производственных проиессов в настоящее время не теряют своей актуальности. Многие существующие устройства и системы работают в ручном или полуавтоматическом режиме, что приводит к известным проблемам. Способы соединения элементов систем управления камнедробильными установками и выбор протоколов передачи сигналов между ними являются достаточно устоявшимися, однако в связи с появлением новых устройств и каналов связи с улучшенными характеристиками вопросы выборов методов и алгоритмов работь подобных систем требуют постоянной модификации. В работе предлагается структура системы управления камнедробильного комплекса. В качестве критерия качества построенной системы используется оценка надежности работы системы при условии выполнения заданных временных ограничений на выполнение основных задач. Элементами новизны представленного решения являются использование оборудования отечественного производства на всех этапах работы системы, а также некоторые решения по выгору каналов связи и протоколов передачи сигналов.

Цель - разработка структуры автоматизированной системь управления камнедробильной установки.

Метод или методология проведения работы: в работе используются методы системного анализа, теории передачи сигналов и теории алгоритмов.

Результаты. Использование представленного решения позволило реализовать более простую и эффективную аппаратную и 
программную модели системы управления по сравнению с аналогами, что позволит уменьшить количество отказов различных подсистем камнедробильной установки. Кроме того, были внесены некоторые новые функиии, такие как возможность удаленного контроля и обмена данными с программами бухгалтерского учета.

Область применения результатов. Полученные результаты целесообразно применять производственными субъектами, осуществляюшими деятельность, одним из элементов которой являются использование дробильных установок и комплексов.

Ключевые слова: АСУ; ПЛК; Моdbus; Овен; камнедробильная установка; контроллер; дробильного комплекс

\section{AUTOMATED CONTROL SYSTEM FOR STONE CRUSHING PLANT}

\section{Myasnikov V.I., Morokhin D.V., Ivanov A.V., Ivanov R.A.}

The issues of automation of production processes are currently not losing their relevance. Many existing devices and systems operate in manual or semi-automatic mode, which leads to known problems. The methods of connecting system elements and the choice of protocols for transmitting signals between them are quite well-established, however, due to the emergence of new devices and communication channels with improved characteristics, the issues of choosing methods and algorithms for the operation of such systems require modification. As a criterion for the quality of the constructed system, an assessment of the reliability of the system is used, provided that the specified time constraints on the performance of the main tasks are met. The elements of novelty of the presented solution are the use of domestically produced equipment at all stages of the system operation, as well as some solutions for the choice of communication channels and signal transmission protocols. Note that the main elements of novelty relate to the software implementation of the system, the description of which 
is beyond the scope of this article and is planned to be published in a separate work.

Purpose. The aim of the work is to develop the structure of an automated control system for a stone crushing plant.

Methodology. The work uses the methods of systems analysis, the theory of signal transmission and the theory of algorithms.

Results. The use of the presented solution made it possible to implement a simpler and more efficient hardware and software model of the control system, which will reduce the number of failures of various subsystems of the stone crushing plant. In addition, some new features have been introduced, such as the ability to remotely control and receiving data to accounting software.

Practical implications. The obtained results are advisable to be applied by production entities carrying out activities, one of the elements of which is the use of crushing plants and complexes.

Keywords: ACS; PLC; Modbus; Aries; stone crushing plant; controller; crushing complex

\section{Введение}

Камнедробильные установки, содержащие различных варианты дробилок, грохотов, конвейеров и вспомогательных подсистем, применяются в различных областях производства [20]. Одним из направлений их использования является получение материалов для дорожно-строительных работ $[4,15,16]$. Вопросы автоматизации работы таких комплексов актуальны не только с точки зрения снижения себестоимости процесса дробления, повышения его производительности, но и для минимизации влияния человеческого фактора на работоспособность установок, улучшения условий труда, улучшения экономических показателей $[1,8,17]$.

На рис. 1 приведена схема дробильного комплекса, где на стадии дробления используется центробежно-ударная дробилка [19]. К вспомогательному оборудованию относятся: питатель, семь конвейеров; два грохота, а также магнит и водяной насос. Питатели и грохота комплектуются вибраторами. Кроме того, используются 
различные датчики (веса, скорости и т.д.) и исполнительные механизмы (устройства аварийного отключения, устройства управления нагрузкой двигателей и т.д.).

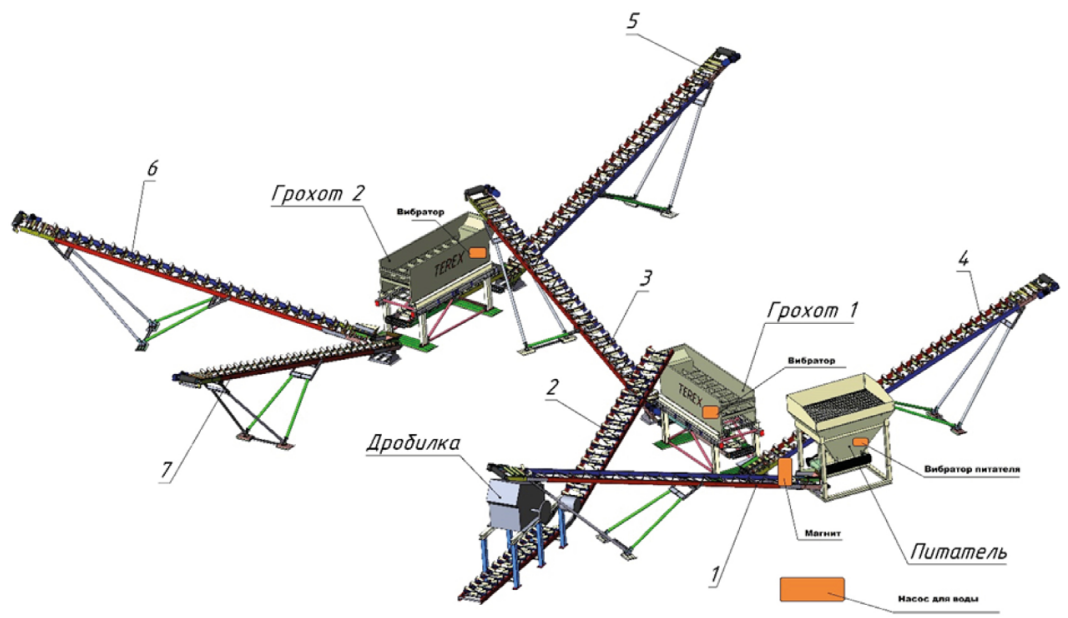

Рис. 1. Схема дробильно-сортировочной линии

Оборудования системы управления:

Для построения системы управления дробильной установкой используется следующее оборудование:

- Программируемый логический контроллер ОВЕН ПЛК11060[M02]

- Сенсорная панель оператора ОВЕН СП315

- Модуль аналогового ввода с универсальными входами OBEH MB110-101

- Преобразователь частоты ОВЕН ПЧВ102-2К2

- Сетевой шлюз для доступа к сервису Owen Cloud ПМ210-24

- Блок питания ОВЕН БП60К для контроллера и панели

- Блок питания ОВЕН БП60Б-Д4-24 для периферийных устройств

Как видно из перечня, используется оборудование отечественного производителя ОВЕН [5]. Данное оборудование опробовано 
во многих подобных системах и является одним из лучших по соотношению цена-качество. Несомненно, существуют системы и элементы других производителей $[7,10,21]$, но их сравнение и выбор не являлись задачей разработчиков, так как перечень оборудования в данном проекте определяется заказчиком.

\section{Структура системы управления}

В общем, структура системы управления имеет следующий вид (рис. 2).

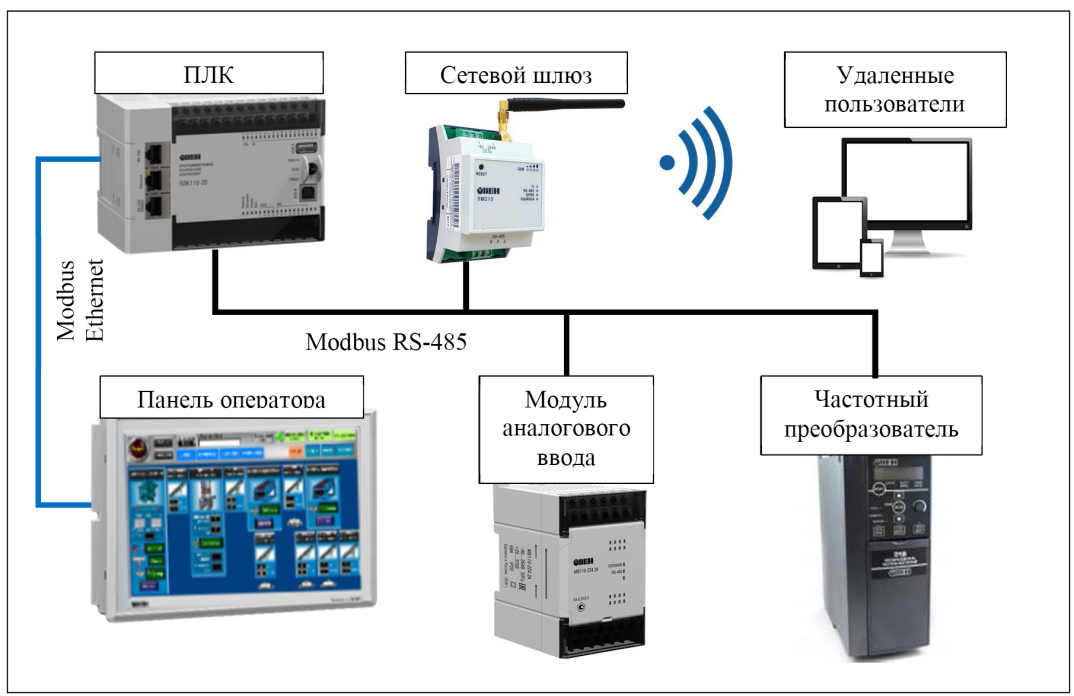

Рис. 2. Структура системы управления

Основным обработчиком информации в системе является ПЛК110-60[М02] [11]. Он производит обработку сигналов от агрегатов системы, поступающих на собственные дискретные входы и аналоговые входы модуля аналогового ввода [9]. Также он выдает управляющие сигналы на собственные дискретные выводы и частотный преобразователь [10], задающий обороты двигателя конвейера питателя. Основным средством взаимодействия с оператором является панель оператора СП315 [11]. Кроме того, для 
удаленного контроля и управления используется сетевой шлюз [12]. Вся обработка ввода и вывод данных также является функцией ПЛК $[6,14]$.

Отметим, что для передачи сигналов внутри системы используются различные среды. Большинство устройств подключены по двухпроводному кабелю и технологии RS-485. Панель оператора связана с контроллером по кабелю Ethernet. Это разделение, кроме удобства подключения, связано с организацией обмена. В связке ПЛК - панель оператора панель является мастером протокола Modbus, а для остальных устройств мастером является ПЛК.

Подобное деление позволяет контроллеру в реальном времени реагировать на изменения состояния управляющих элементов панели. Если панель работает в режиме slave, то ПЛК вынужден отслеживать состояние панели через заданные временные промежутки (poll time) либо изменения значений (value). Это может вызывать задержки, влекущие за собой неоднозначность интерпретации введенных данных. Например, быстрое двойное нажатие кнопки может обработаться как одинарное, что приведет к рассогласованию состояния панели и соответствующих данных ПЛК.

Если же панель находится в режиме мастер (master), то все изменения непосредственно вызывают обновление регистров ПЛК и, соответственно, обрабатываются программой.

Для остальных элементов системы такой режим необязателен (и сложнореализуем при заданных условиях), так как они работают либо только в режиме чтения, либо в режиме записи.

Также в системе работает сетевой шлюз, обеспечивающий связь по радиоканалу (технология GPRS) с внешними пользователями для удаленного мониторинга, управления и сбора статистики.

\section{Интерфейс пользователя}

По согласованию с заказчиком был разработан интерфейс пользователя, реализованный на панели оператора СП315 [18] (рис. 3).

Реализованы два основных режима работы: «Работа» и «Наладка». Их основное отличие в том, что режим «Наладка» позволяет 
включить оборудование в любой последовательности. Режим «Работа» определяет четкую последовательность включения агрегатов. Согласно техническому заданию, очередной агрегат должен включаться не автоматически, а при нажатии кнопки запуска оператором, если подошла очередь согласно порядку включения. При этом, если через заданный промежуток времени не будет получен сигнал, подтверждающий запуск агрегата, система переходит в состояние ошибки. Далее, в зависимости от заданных условий, система останавливается полностью, либо частично.

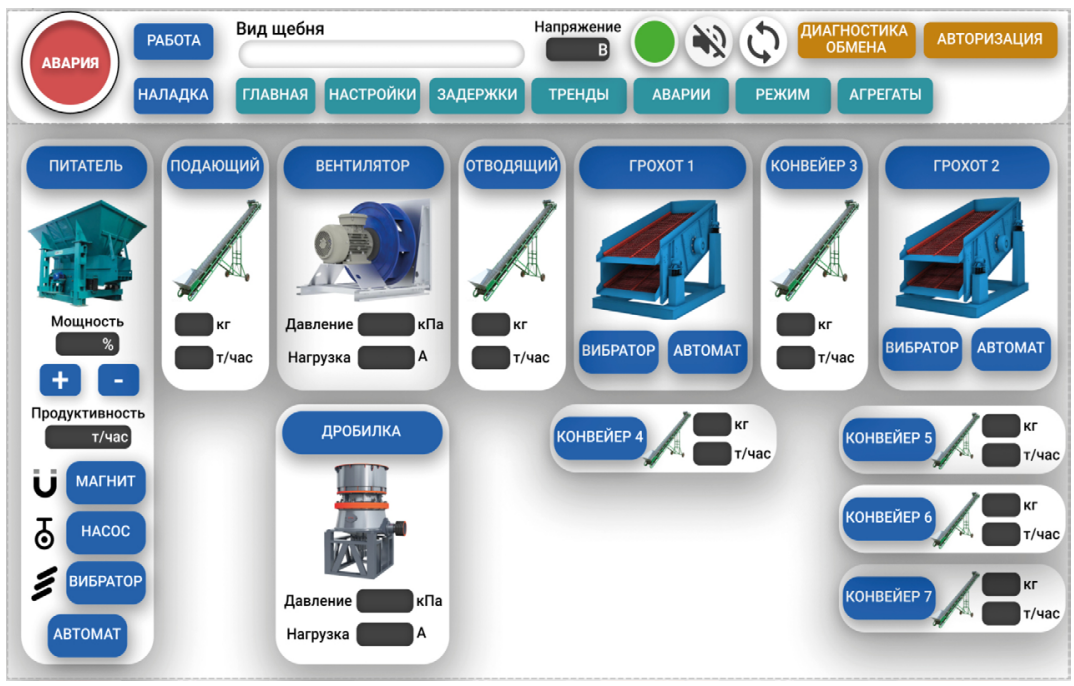

Рис. 3. Главный экран системы управления

Очередной агрегат для запуска помечается определенной подсветкой. Некоторые элементы могут включаться независимо от других после полного запуска системы. Например, насос, вибраторы, магнит и т.п. Вибраторы также могут работать в автоматическом режиме, включаясь через заданные периоды времени.

Кроме того, на экран выводится информация, полученная с датчиков системы, таких как подтверждение работы агрегата, текущие данные по нагрузке, производительности и т.п. 
Вспомогательные окна дают возможность выполнить дополнительные настройки (задержек, критических значений, ...) или просмотреть заданную статистику по работе системы и ошибкам.

Также предусмотрена защита некоторых режимов и данных посредством паролей.

В качестве дополнительной возможности работы с системой, особенно при настройке и возникновении неисправностей, органы управления дублируются в системе программирования, через интерфейс Codesys HMI (система исполнения визуализаций) [2].

В этом режиме появляются дополнительные возможности по настройке оборудования, калибровке агрегатов и другие сервисные функции.

\section{Выводы}

Таким образом, в статье описана структура разработанной системы управления камнедробильной установкой. Её основными особенностями являются использование отечественной аппаратной базы и разделение устройств по группам с различными протоколами и форматами обмена.

Наиболее интересные и новые решения имеет программная часть системы. Ее описание будет выполнено в отдельной статье. Некоторые особенности отражены в работе [3].

Данная структура была опробована на объекте в тестовом режиме и показала свою работоспособность. После проведения испытаний в реальном режиме работы будет проведена ее оценка и, при необходимости, доработка. Также планируется публикация практических результатов внедрения системы.

Еще одним важным результатом можно считать возможность использования результатов работы в учебном процессе как на строительных направлениях подготовки, например 08.03.01 Автомобильные дороги, так и на информационных, таких как 09.03.01 Информатика и вычислительная техника, 27.03.04 Управление и информатика в технических системах. 


\section{Список литературы}

1. Банников Е.В. Использование ПЛК в промышленности // International scientific review of the problems and prospects of modern science and education. 2019. №LV. C. 25-28.

2. Визуализация CodeSys. Дополнение к руководству пользователя по программированию ПЛК в CodeSys 2.3. 3S - Smart Software Solutions GmbH Memminger Straße 151, 2008.

3. Иванов А.В., Иванов Р.А., Мясников В.И. Особенности визуализации в Codesys 2.3 // Инженерные кадры - будущее инновационной экономики России: материалы VI Всероссийской студенческой конференции (Йошкар-Ола, 10-13 ноября 2020 г.): Часть 4: Информационные технологии - основа стратегического прорыва в современной промышленности. Йошкар-Ола: Поволжский государственный технологический университет, 2020. 217 с.

4. Калмыков Б.М., Васильев Ю.Г. Автоматизация управления дорожно-строительным предприятием // Вестник ЧГУ. 2005. №2. C. 205-211.

5. Компания ОВЕН. https://owen.ru

6. Максимычев О.И. Программирование логических контроллеров (PLC): учеб. пособие / О.И. Максимычев, А.В. Либенко, В.А. Виноградов. М.: МАДИ, 2016. 188 с.

7. Минаев И.Г. Программируемые логические контроллеры. Практическое руководство для начинающего инженера / И. Г. Минаев, В. В. Самойленко. Ставрополь: АГРУС, 2009. 100 с.

8. Минаев И.Г. Свободно программируемые устройства в автоматизированных системах управления / И.Г. Минаев, В.В. Самойленко, Д.Г. Ушкур, И.В. Федоренко. Ставрополь: АГРУС. 2016. 168 с.

9. Модули аналогового ввода с универсальными входами (с интерфейсом RS-485) MB110. https://owen.ru/product/moduli_analogovogo_ vvoda_s_universal_nimi_vhodami_s_interfejsom_rs_485

10. Мустаев А.Ф. Сравнительный анализ рыночных моделей ПЛК // Электрооборудование: эксплуатация и ремонт. 2020. № 11. С. 44-46.

11. ПЛК110 [M02] контроллер для средних систем автоматизации с DI/DO (обновленный). https://owen.ru/product/plk110_m02 
12. ПМ210 сетевой шлюз для доступа к сервису OwenCloud RS-485. https://owen.ru/product/pm210

13. ПЧВ2 преобразователь частоты. https://owen.ru/product/preobrazovatel_ chastoti_oven_pchv1_i_pchv2

14.Руководство пользователя по программированию ПЛК в CoDeSys 2.3. 3S Smart Software Solutions GmbH Memminger Straße. 151, 2008.

15.Салихов М.Г. Проектирование и организация работ на асфальтобетонных и цементобетонных заводах Методические указания к выполнению курсовой работы. Йошкар-Ола: ПГТУ, 2014. $20 \mathrm{c}$.

16. Салихов М.Г. Проектирование и организация работы карьера, камнедробильного, асфальтобетонного и цементобетонного заводов: учебное пособие. Йошкар-Ола: Поволжский государственный технологический университет, 2016. 60 c.

17. СП 12-136-2002 «Решения по охране труда и промышленной безопасности в проектах организации строительства и проектах производства работ».

18. СПЗхх сенсорные панели оператора. https://owen.ru/product/sp3xx.

19.Центробежное устройство дробления (ЦУД) - 1600. http:// drobtehmash.ru/tsentrobezhnoye-ustroystvo-drobleniya-tsud-1600

20. Чебан А.Ю., Хрунина Н.П. Классификация дробильных установок и технологических схем их применения при переработке строительных горных пород // Системы. Методы. Технологии. 2016. №1. C. 98-103.

21. Щербаков К.С., Щербаков С.А., Кочегуров А.И. Разработка встроенного программного обеспечения для программируемых логических контроллеров, используемых в области промышленной автоматизации // Известия ТПУ. 2009. №5. С. 28-32.

\section{References}

1. Bannikov E.V. Ispol'zovanie PLK v promyshlennosti [PLC use in industry]. International scientific review of the problems and prospects of modern science and education, 2019, №LV, pp. 25-28. 
2. Vizualizatsiya CodeSys. Dopolnenie k rukovodstvu pol'zovatelya po programmirovaniyu PLK v CodeSys 2.3. 3S - Smart Software Solutions GmbH Memminger Straße 151, 2008.

3. Ivanov A.V., Ivanov R.A., Myasnikov V.I. Osobennosti vizualizatsii v Codesys 2.3 [Features of visualization in Codesys 2.3]. Inzhenernye kadry - budushchee innovatsionnoy ekonomiki Rossii: materialy VI Vserossiyskoy studencheskoy konferentsii (Yoshkar-Ola, 10-13 noyabrya 2020 g.): Chast' 4: Informatsionnye tekhnologii - osnova strategicheskogo proryva $v$ sovremennoy promyshlennosti [Engineering personnel - the future of the innovative economy of Russia: materials of the VI All-Russian student conference (Yoshkar-Ola, November 10-13, 2020): Part 4: Information technology - the basis of a strategic breakthrough in modern industry]. Yoshkar-Ola: Volga State Technological University, 2020, 217 p.

4. Kalmykov B.M., Vasil'ev Yu.G. Avtomatizatsiya upravleniya dorozhno-stroitel'nym predpriyatiem [Automation of management of a road-building enterprise]. Vestnik ChGU, 2005, no. 2, pp. 205-211.

5. OVEN company. https://owen.ru

6. Maksimychev O.I., Libenko A.V., Vinogradov V.A. Programmirovanie logicheskikh kontrollerov (PLC) [Programming logic controllers (PLC)]. M.: MADI, 2016, 188 p.

7. Minaev I.G., Samoylenko V.V. Programmiruemye logicheskie kontrollery. Prakticheskoe rukovodstvo dlya nachinayushchego inzhenera [Programmable logic controllers. A practical guide for a novice engineer]. Stavropol': AGRUS, 2009, 100 p.

8. Minaev I.G., Samoylenko V.V., Ushkur D.G., Fedorenko I.V. Svobodno programmiruemye ustroystva $v$ avtomatizirovannykh sistemakh upravleniya [Freely programmable devices in automated control systems]. Stavropol': AGRUS, 2016, 168 p.

9. Modules of analog input with universal inputs (with RS-485 interface) MV110. https://owen.ru/product/moduli_analogovogo_vvoda_s_universal_nimi_vhodami_s_interfejsom_rs_485

10. Mustaev A.F. Sravnitel'nyy analiz rynochnykh modeley PLK [Comparative analysis of PLC market models]. Elektrooborudovanie: ekspluatatsiya i remont, 2020, no. 11, pp. 44-46. 
11. PLC110 [M02] controller for medium automation systems with DI/ DO (updated). https://owen.ru/product/plk110_m02

12.PM210 setevoy shlyuz dlya dostupa k servisu OwenCloud RS-485. https://owen.ru/product/pm210

13. PChV2 frequency converte. https://owen.ru/product/preobrazovatel_ chastoti_oven_pchv1_i_pchv2

14. User manual for PLC programming in CoDeSys 2.3. 3S Smart Software Solutions GmbH Memminger Straße. 151, 2008.

15. Salikhov M.G. Proektirovanie i organizatsiya rabot na asfal tobetonnykh i tsementobetonnykh zavodakh Metodicheskie ukazaniya k vypolneniyu kursovoy raboty [Design and organization of work at asphalt and cement concrete plants Methodical instructions for the implementation of term paper]. Yoshkar-Ola: PSTU, 2014, $20 \mathrm{p}$.

16. Salikhov M.G. Proektirovanie i organizatsiya raboty kar'era, kamnedrobil'nogo, asfal tobetonnogo i tsementobetonnogo zavodov: uchebnoe posobie [Design and organization of work of a quarry, stone crushing, asphalt and cement concrete plants: a tutorial]. Yoshkar-Ola: Volga State Technological University, 2016, $60 \mathrm{p}$.

17. SP 12-136-2002 "Solutions for labor protection and industrial safety in construction management projects and work production projects".

18. SP3xx touchscreen operator panels. https://owen.ru/product/sp3xx.

19. Centrifugal crushing device -1600 . http://drobtehmash.ru/tsentrobezhnoye-ustroystvo-drobleniya-tsud- 1600

20. Cheban A.Yu., Khrunina N.P. Klassifikatsiya drobil'nykh ustanovok i tekhnologicheskikh skhem ikh primeneniya pri pererabotke stroitel'nykh gornykh porod [Classification of crushing plants and technological schemes of their use in the processing of building rocks]. Sistemy. Metody. Tekhnologii, 2016, no. 1, pp. 98-103.

21. Shcherbakov K.S., Shcherbakov S.A., Kochegurov A.I. Razrabotka vstroennogo programmnogo obespecheniya dlya programmiruemykh logicheskikh kontrollerov, ispol'zuemykh v oblasti promyshlennoy avtomatizatsii [Development of embedded software for programmable logic controllers used in the field of industrial automation]. Izvestiya $T P U, 2009$, no. 5, pp. 28-32. 


\section{ДАННЫЕ ОБ АВТОРАХ}

Мясников Владимир Иванович, доцент кафедры Информационно-вычислительных систем, кандидат технических наук ФГБОУ ВО «Поволжский государственный технологический университет»

Площзадь Ленина, 3, г. Йошкар-Ола, Республика Марий Эл, 424000, Российская Федерация

MyasnikovVI@volgatech.net

Морохин Дмитрий Витальевич, заведующий кафедрой ИВС, кандидат технических наук ФГБОУ ВО «Поволжский государственный технологический университет»

Площзадь Ленина, 3, г. Йошкар-Ола, Республика Марий Эл, 424000, Российская Федерация dvmitry@mail.ru

Иванов Артем Владимирович, студент группы ИВТ-21 ФГБОУ ВО «Поволжский государственный технологический университет»

Площзадь Ленина, 3, г. Йошкар-Ола, Республика Марий Эл, 424000, Российская Федерация wladimirovichai@gmail.com

Иванов Роман Андреевич, студент группы ИВТ-21

ФГБОУ ВО «Поволжский государственный технологический университет»

Площзадь Ленина, 3, г. Йошкар-Ола, Республика Марий Эл, 424000, Российская Федеращчи verdenum@gmail.com

\section{DATA ABOUT THE AUTHORS}

Vladimir I. Myasnikov, Associate Professor of the Department of IVS with the title of Associate Professor Volga State Technological University 
3, Lenin Square, Yoshkar-Ola, Mari El, 424000, Russian Federation

MyasnikovVI@marstu.net

Dmitry V. Morokhin, Head of the Department of IVS with the title of Associate Professor Volga State Technological University

3, Lenin Square, Yoshkar-Ola, Mari El, 424000, Russian Federation

dvmitry@mail.ru

Artem V. Ivanov, student

Volga State Technological University

3, Lenin Square, Yoshkar-Ola, Mari El, 424000, Russian Federation

wladimirovichai@gmail.com

Roman A. Ivanov, student

Volga State Technological University

3, Lenin Square, Yoshkar-Ola, Mari El, 424000, Russian Federation

verdenum@gmail.com 